\title{
Bridging the Gap between $\mathrm{H}$ - and J-Aggregates: Classification and
}

\section{Supramolecular Tunability for Excitonic Band Structures in 2-Dimensional}

\section{Molecular Aggregates}

Arundhati P. Deshmukh, ${ }^{1}$ Niklas Geue, ${ }^{1,2}$ Nadine C. Bradbury, ${ }^{1}$ Timothy L. Atallah, ${ }^{1,3}$ Chern Chuang, ${ }^{4}$ Monica Pengshung, ${ }^{1}$ Jianshu Cao, ${ }^{5}$ Ellen M. Sletten, ${ }^{1}$ Daniel Neuhauser,${ }^{1}$ and Justin R. Caram ${ }^{1 *}$

${ }^{1}$ Department of Chemistry and Biochemistry, University of California, Los Angeles, 607 Charles E. Young Dr. East, Los Angeles, California 90095, United States. ${ }^{2}$ The Michael Barber Centre for Collaborative Mass Spectrometry, Manchester Institute of Biotechnology, The University of Manchester, Oxford Road, Manchester M13 9PL, United Kingdom. ${ }^{3}$ Department of Chemistry and Biochemistry, Denison University, 500 West Loop, Granville, Ohio 43023, United States. ${ }^{4}$ Department of Chemistry, University of Toronto, Toronto, Ontario, Canada. ${ }^{5}$ Department of Chemistry, Massachusetts Institute of Technology, Cambridge, Massachusetts 02139, United States.

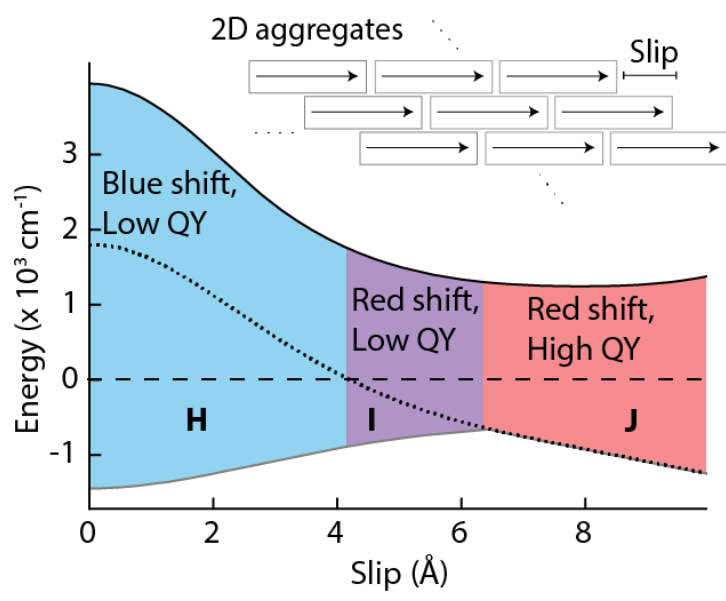

Abstract: Molecular aggregates with long-range excitonic couplings have drastically different photophysical properties compared to their monomer counterparts. From Kasha's model for 1-dimensional systems, positive or negative excitonic couplings lead to blue or red shifted optical spectra with respect to the monomers, labelled $\mathrm{H}$-and J-aggregates respectively.

The overall excitonic couplings in higher dimensional systems are much more complicated and cannot be simply classified from their spectral shifts alone. Here, we provide a unified classification for extended 2D aggregates using temperature dependent peak shifts, thermal broadening and quantum yields. We 
discuss the examples of six 2D aggregates with J-like absorption spectra but quite drastic changes quantum yields and superradiance. We find the origin of the differences is, in fact, a different excitonic band structure where the bright state is lower energy than the monomer but still away from the band edge. We call this an 'I-aggregate'. Our results provide a description of the complex excitonic behaviors that cannot be explained solely on Kasha's model. Further, such properties can be tuned with the packing geometries within the aggregates providing supramolecular pathways for controlling them. This will allow for precise optimizations of aggregate properties in their applications across the areas of optoelectronics, photonics, excitonic energy transfer, and shortwave infrared technologies.

\section{Introduction}

Excitonically coupled molecular aggregates are materials derived from chromophores whose optoelectronic behavior changes upon aggregation due to collective interactions of monomer transition dipole moments (TDMs). Such aggregates are represented in many natural and synthetic materials, including conjugated polymers, supramolecular chromophore assemblies and photosynthetic complexes. ${ }^{1,2}$ Extended aggregates of chromophores have found applications in many areas - energy transfer, ${ }^{1,3,4}$ polaritonics or plexitonics, ${ }^{5-8}$ a variety of optoelectronic devices such as luminescent solar concentrators, OLEDs, ${ }^{9,10}$ and shortwave infrared technologies (SWIR, 1000-2000 nm). ${ }^{11-13}$ In extended aggregate systems, the huge enhancements of TDMs from monomers to aggregates result in excitonic superradiance, wherein a large number of in-phase TDMs lead to collective emission phenomena. Superradiance can be useful in modulating exciton transport and telecommunication related applications. ${ }^{13,14}$ These applications can require high quantum yields and, and efficient energy/charge transfer, which in turn directly depend on the underlying exciton band structure. Therefore, elaborating structure-property relationships for the excitonic band structures will allow for supramolecular tunability of such properties, enabling rational design of molecular aggregates. 
In the 1950s, Kasha developed a framework to relate the excitonic shifts in the optical spectra of molecular aggregates to the underlying structure. Within this framework, when the transition dipoles are arranged head-to-tail, the excitonic state with all dipoles oscillating in-phase (with the wavevector $k=0$ ) is lowered in energy compared to the monomer. This leads to a red shift in the optical transitions. 1D excitonic systems with such head-to-tail arrangements are called J-aggregates. ${ }^{15}$ This $k=0$ state is the 'bright state' as it has the highest transition probability based on Fermi's golden rule. ${ }^{2}$ Conversely, cofacial arrangement leads to blue shifted transitions as the bright state is higher in energy than the monomer, known as H-aggregates. All other arrangements result in Davydov splitting with two dipoles per unit cell, producing two excitonic peaks for any single set of electronic transitions. ${ }^{16,17} \mathrm{~J}$-aggregates are typically characterized by enhanced quantum yields from their respective monomers, high radiative rates and narrow linewitdths. ${ }^{1,18}$ In cases where the net TDM is enhanced compared to the monomer, Jaggregates can show excitonic superradiance. $\mathrm{H}$-aggregates, on the other hand, usually have low quantum yields as non-radiative relaxation to the dark band edge excitonic state dominates the excited state decay. $^{1}$

Kasha's model arises from considering only dimers and 1-dimensional chains as well as nearest neighbor dipolar interactions. These limitations lead to the model's failure in describing systems with permanent dipole moments, ${ }^{19}$ charge transfer or quadrupolar excitonic coupling, ${ }^{20}$ or higher dimensional aggregates, e.g. sheet-like and tubular aggregates. ${ }^{21-23}$ Examples of non-Kasha behavior have also been observed in polydiacetylene, poly(3-hexylthiophene) and other conjugated polymers, referred to as segregated "HJ" aggregates, wherein interchain and intrachain couplings have opposite signs. ${ }^{24,25}$ In some cases, different forms of couplings can cancel out, resulting in a null aggregate with no excitonic shift in optical spectra in spite of a significant excitonic bandwidth. ${ }^{26,27}$

Using the Frenkel exciton model with only long-range dipole-dipole couplings, ${ }^{28}$ we have previously shown the presence of 'I-aggregates' in 2D lattices. ${ }^{22}$ We define l-aggregates with an excitonic band where 


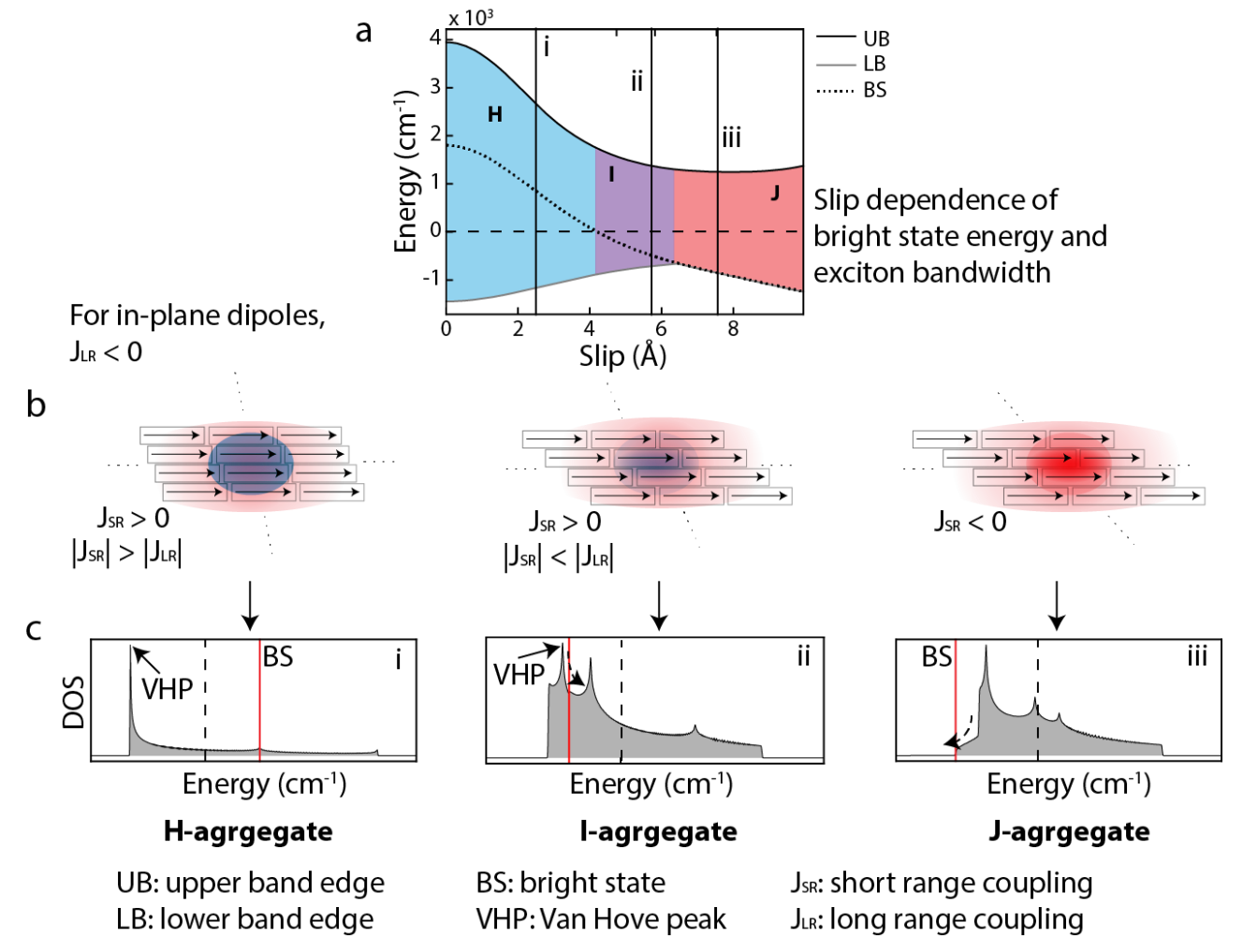

Figure 1. a. Bandwidth of the exciton DOS and position of the bright state (dotted line) as a function of slip in 2dimensional (2D) aggregates, dashed line indicates monomer energy. Blue region: bright state higher in energy than monomer ( $\mathrm{H}$-aggregate), purple region: bright state lower in energy than monomer but away from the lower band edge (I-aggregate), and red region: bright state lower in energy than the monomer and at the band edge (Jaggregate), b. schematic showing possibilities of short and long range couplings for $2 \mathrm{D}$ aggregates with in-plane dipoles leading to $\mathrm{H}-, \mathrm{I}$ - and J-aggregates, c. representative excitonic DOS for slips corresponding to the vertical lines $\mathrm{i}$, ii and iii on part a showing $\mathrm{H}-, \mathrm{I}$ - and J-aggregate band structures respectively. Solid red line indicates bright state energy and dashed line indicates monomer energy. Curved dashed arrows indicate the direction of temperature dependent shifts.

the bright state is red shifted from the monomer but still has dark states below it, drawing characteristics from both $\mathrm{H}$ - and J-aggregates. We showed that such behaviors depend upon the relative slip between adjacent monomers in the 2D lattices (Figure 1a). I-aggregates have red shifted aggregate to monomer absorption (J-like) and yet typically suppressed quantum yields (H-like). ${ }^{22} \mathrm{~J}$-aggregates, on the other hand, should have a bright state at the band edge (or within $k_{B} T$ from the band edge where $k_{B}$ is Boltzmann constant). Therefore, J-aggregates will have enhanced radiative rates with respect to the monomers, which further increase at low temperatures. J-aggregates will also show persistent excitonic superradiance due to simultaneous increase in quantum yields and reduced excited state lifetimes. Since 
I- and J-aggregates both show aggregate to monomer red shifts, a more comprehensive classification system for 2D aggregates is needed. Here, we demonstrate the distinct possibilities of excitonic band structures in 2D aggregates and their experimental signatures. Pathways for chemically tuning these excitonic band structures are necessary to access a breadth of photophysical properties in molecular aggregates as well as to optimize material properties for specific applications. For example, chemically tuning the band structures from a non-band edge I-aggregate to band edge J-aggregate will enable aggregates with high quantum yields, a prerequisite for many applications. ${ }^{29,30}$

We start with a detailed discussion of the theoretical basis for excitonic band structures of 2D aggregates provided by Chaung et al. ${ }^{31}$ The positive or negative contributions of short and long range couplings lead to critical differences in the band structures which has distinct spectroscopic signatures in emission and temperature dependent properties. Based on this, we provide a classification system for 2D aggregates based on aggregate to monomer shifts, temperature dependent peak shifts and broadening, as well as quantum yield enhancements. We measure quantum yields, lifetimes, superradiance, temperature dependent peak shifts and full-width at half maximum (FWHM) across a set of 2D aggregates of six closely related cyanine dyes. Stochastic Hamiltonian modelling allows for rapid screening of the large parameter space for aggregate geometries and disorders, relating the observed photophysical properties to the underlying geometries. We show that such differences in the band structures and ultimately, the photophysical properties, can be manipulated with the supramolecular packing of the chromophores within the extended 2D structures. The relative slip between adjacent monomers as well as length of the monomer determine the relative contributions of short and long range couplings, which in turn, result in the distinct excitonic band structures of $\mathrm{H}-, \mathrm{I}$ - and J-aggregates. This leads to an internally consistent description of the distinct photophysical behaviors - enhanced or suppressed quantum yields relative to their monomers, differences in superradiance and red or blue shifts with temperature. Our findings can 
be utilized to rationally design molecular excitonic systems with tunable photophysical properties, enabling optimization of material properties for specific applications.

\section{Results and Discussion}

We calculate the excitonic density of states (DOS) and the bright state energy using the Frenkel exciton Hamiltonian with brick layer model. Within this model, we treat each monomer as a brick with a given dipole moment $(\mu)$, length $(L)$, width $(w)$, and tile them in a 2D brick layer lattice with different slips (s). ${ }^{22,28,32}$ The eigenvalues of this Hamiltonian provide the excitonic DOS and Fermi's golden rule gives the bright state. See supplemental information (SI) computational methods and Figure S1 for details. Table S1 lists the parameters used in the model. In Figure 1a, we plot the bright state, upper and lower band edge of the DOS for a 2D brick layer aggregate with varying slip values. As we increase the slip, the band structure goes from $\mathrm{H}$-aggregate (bright state blue shifted from monomer), to l-aggregate (bright state red shifted but away from the band edge) and finally to J-aggregate (bright state at the lower band edge).

Significance of short and long range couplings: Prior theory by Chuang et al. explains the origin of $\mathrm{H}-$, I- and J-aggregate behaviors on the basis of the types of short and long range couplings in 2D aggregates. ${ }^{31}$ The possible short and long range couplings for a 2D aggregate with in-plane TDMs are explained in Figure 1b. Long-range interactions in extended 2D systems with in-plane TDMs always contribute a net negative coupling $\left(J_{L R}<0\right)$. Short range interactions can either be positive or negative depending on the relative slip, orientation and the coupling model. In cases where the short-range couplings are positive and higher in magnitude than the long range couplings, we obtain a 2D H-aggregate. All slip values in the blue region of Figure 1a show this behavior. In Figure 1c, we show a representative example of the excitonic DOS for a slip value denoted with roman numeral ' $i$ ' on Figure 1 a. Since there are always some negative contributions in $2 \mathrm{D}$ lattices, the bright state is not at the upper band edge. ${ }^{31}$ When the short-range 
coupling is positive, but smaller than the long-range coupling, the bright state is redshifted from the monomer but still has lower energy dark states below, making it an I-aggregate. The overall coupling, in this case, is negative resulting in red shifted aggregate to monomer absorptions. This is denoted by purple shaded region on Figure 1a and a representative DOS is shown in Figure 1c corresponding to the slip denoted by roman numeral 'ii'. For a 2D J-aggregate, both the short and long range couplings are negative, making the bright state also the lowest energy state in the excitonic DOS, similar to Kasha's model. This scenario is shown in red shaded region on Figure 1a and a representative DOS in Figure 1c (denoted by 'iii').

As shown in Figure 1c, the 2D excitonic DOS has several sharp features, or Van Hove Peaks (VHPs). VHPs arise from extrema in the dispersion relations where the dispersion curve is flat i.e. $\frac{d E}{d k}=0$ (in the 2D case, the extrema are saddle points in the dispersion curves). Effectively, a VHP consists of a large number of states concentrated in a close energy range. In other 2D materials (e.g. graphene), this is known to cause superconductivity, topological insulators and other exotic phenomena. ${ }^{33-35}$ In case of the molecular aggregates, position of the bright state relative to the VHPs can be useful in distinguishing Iaggregates from J-aggregates and ultimately, explaining many of the photophysical properties such as quantum yields, linewidths, temperature dependent shifts and transport properties.

Chuang et al. also provide relationships between the position of bright state relative to the VHP and temperature dependent peak shifts. ${ }^{31}$ For bright states lower in energy than the VHP, the local slope of the DOS is positive meaning that there are more dark states on the higher energy side of the bright state than on the lower energy side. With increasing temperature, this asymmetric distribution of the dark states near the bright state pushes the bright state away towards lower energy, resulting in a red shift. Thus, the positive slope near the bright state results in a red shift with increasing temperature (indicated by dashed arrows on Figure 1c). Conversely, for bright states higher in energy than the VHP, the local slope 
of the DOS is negative with relatively higher number of states below the bright state and thus, we observe a temperature dependent blue shift.

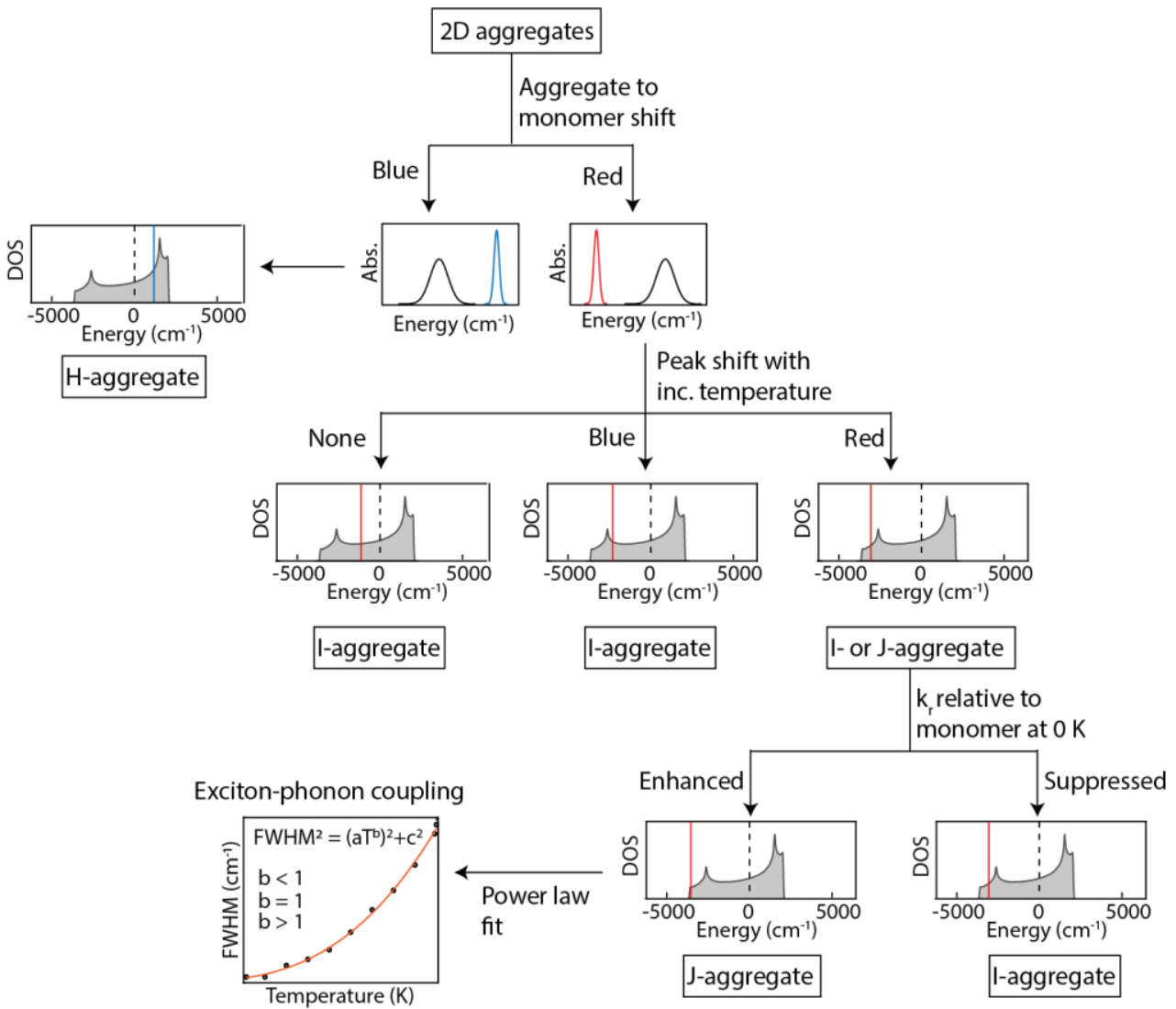

Figure 2. Schematic of the classification scheme for 2-dimensional excitonic aggregates based on aggregate to monomer shifts, temperature dependent shifts and radiative rate $\left(k_{r}\right)$ enhancement from the monomers. Dashed vertical lines indicate monomer energy and vertical colored lines indicate bright states.

Classification scheme for 2D aggregates: Such distinct possibilities in 2D aggregates suggest that excitonic peak shifts alone are not diagnostic of the band structure, coupling strength, and emissivity. To probe higher dimensional aggregate structures, one must go beyond aggregate to monomer absorption peak shifts and narrowing. Expanding on previous theories, ${ }^{22,31}$ we present a classification system encompassing such diverse excitonic behaviors in 2D aggregates based on peak shifts and peak broadening with temperature as well as room temperature quantum yields. The following paragraph and Figure 2 summarize our classification system: 
For 2D aggregates, a blue shifted absorption from aggregate to monomer indicates $\mathrm{H}$-aggregation. Whereas, for aggregates with red shifted absorptions from their monomers, both I- and J-aggregation is possible. The position of the bright state with respect to the VHPs contributes to the temperature dependent properties. For band-edge J-aggregates, the local slope of the DOS is always positive. Therefore, band-edge J-aggregates must show red shifts with increasing temperature, whereas Iaggregates can show red, blue or no shifts with temperature depending on the local slope (positive, negative or zero, respectively) of the DOS near the bright state. As a result, a blue or not shifting aggregate peak with temperature can be a conclusive evidence for an I-aggregate. Temperature dependent red shift along with enhanced radiative rate from the monomer can be a conclusive evidence for J-aggregates.

Changes in quantum yields with temperature can also be related to I- or J-aggregation behavior based on thermal occupation of the bright state. In band-edge J-aggregates, one expects a decreasing quantum yield upon heating as the occupation number of the lowest energy bright state will decrease with temperature. For non-band edge I-aggregates, higher temperatures enable occupation of higher energy states leading to increasing quantum yield upon heating. For J-aggregates, temperature dependent power laws can inform on exciton-phonon coupling processes that lead to thermal broadening (discussed in detail later). Further, the amount of thermal broadening can also be used as a relative measure of the total distance of the bright state from the band edge (also discussed in detail later). ${ }^{22}$ Overall, these properties form a complete description of the distinct behaviors possible in 2D excitonic systems, where each experimental observable (quantum yields, temperature dependent shifts and broadening, power laws) can be related to a specific aspect of the excitonic band structure.

We demonstrate examples of the distinct excitonic band structures across $2 \mathrm{D}$ aggregates of six dyes with different polymethine bridge lengths $(\mathrm{Cy} 3, \mathrm{Cy} 5$ and $\mathrm{Cy} 7 ; \mathrm{Cy}=$ cyanine $)$ and substitutions on the central position, shown in Figure 3a. In our earlier work, thermodynamic control of the aggregate selfassembly enabled selective stabilization of each aggregate morphology via an interplay of concentration, 
a
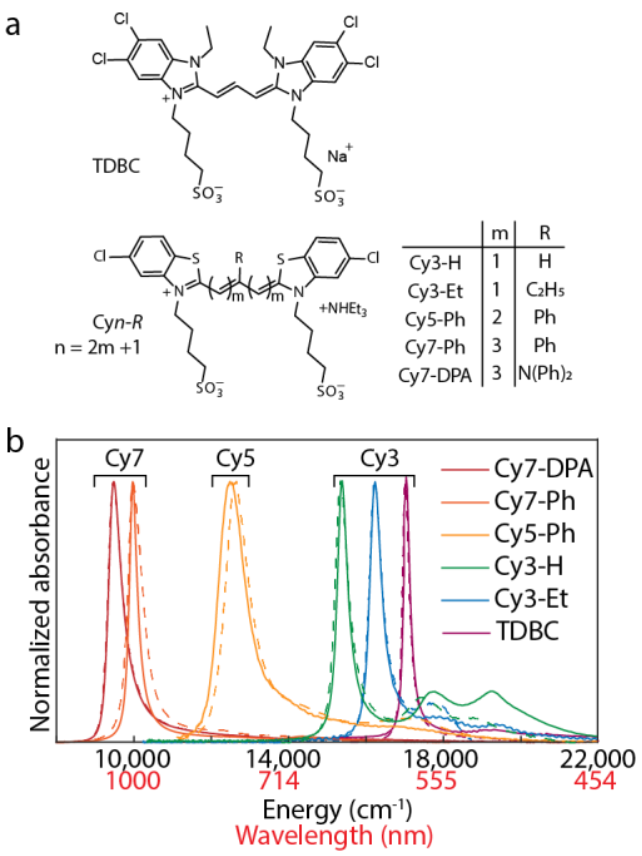

Figure 3. a. Structure of the six dyes explored in this work - benzimidazole cyanine dye TDBC and benzothiazole cyanine with varying central position subsitutions and bridge lengths, $b$. Absorption spectra of all the aggregates in solutions (solid lines) and dried 1:1 (w/w) sucrose:trehalose sugar matrix (dashed lines). All aggregate solutions are in water-methanol or aq. $\mathrm{NaCl}$-methanol mixtures.

solvent:non-solvent ratio, and dielectric screening. Using this approach, we were able to stabilize the 2D sheet-like morphology of all the dyes studied here (SI Figure S2). ${ }^{36}$ We also include the well-known dye 3,3'-bis(4-sulfobutyl)-5, 5',6,6'-tetrachloro-1,1'-diethylbenzimidacarbocyanine, sodium salt (TDBC), commonly used in photonic applications. ${ }^{37,38}$ All $2 \mathrm{D}$ aggregates are prepared by injecting a methanol solution of the dye in water or aqueous $\mathrm{NaCl}$ (see experimental section for exact conditions), followed by equilibration in the dark for $24 \mathrm{~h}$. By changing the dye lengths as well substitutions on the central position of the cyanine bridge (Figure 3a), we afford extended 2D aggregates with different brick lengths and slips. As shown in Figure 1 and pervious works, these parameters lead to the subtle variations in excitonic band structure. ${ }^{23,31}$ The absorptions of these $2 \mathrm{D}$ aggregates range from $587 \mathrm{~nm}$ (TDBC) to $1050 \mathrm{~nm}$ (Cy7-DPA), shown in Figure $3 \mathrm{~b}$. The narrow lineshape with a high energy tail observed across all aggregates is characteristic of extended 2D aggregate structures, and is also seen in other 2D aggregates of perylene bisimides. ${ }^{39}$ 
Quantum yield and superradiance: We measure the emissive properties of all the aggregates at room temperature (RT). We highlight TDBC as an archetypal example in Figure 4 owing to the narrowest absorption linewidth (Figure 3b) and highest quantum yield of TDBC aggregate (discussed later in Table 1). As seen from the solution absorption and emission spectra in Figure 4a, TDBC aggregate (red) has narrowed absorption and emission compared to the monomer (blue), and very small Stokes shift ( $2 \mathrm{~nm})$ in the aggregate form. ${ }^{1}$ Figure 4 a inset shows the time-correlated single photon counting (TCSPC) histograms for both the monomer and aggregate of TDBC. Absolute quantum yields and lifetimes in RT

Table 1. Emissive properties of all the dye monomers and 2D aggregates in solutions at room temperature. Lifetimes $(\tau)$, quantum yields $(\phi)$, and transiton dipole moments $(\mu)$ for all monomers (subscript mon) and aggregates (subscript $a g g$ ) along with superradiance rates $\left(\eta_{S R}\right)$ calculated using eq. (1) and eq. (2) are shown. Errors on quantum yields are standard deviations across 3 samples.

\begin{tabular}{|c|c|c|c|c|c|c|c|}
\hline Dye & $\tau_{\text {mon }}$ (ps) & $\begin{array}{c}\boldsymbol{\tau}_{\text {agg }} \\
\text { (ps) }\end{array}$ & $\boldsymbol{\Phi}_{\text {mon }}$ (\%) & $\boldsymbol{\Phi}_{\text {agg }}$ (\%) & $\begin{array}{c}\boldsymbol{\mu}_{\text {mon }} \\
\text { (D) }\end{array}$ & $\mu_{\text {agg }}$ (D) & $\eta_{\boldsymbol{S R}}$ \\
\hline TDBC & 80 & 150 & $2.8 \pm 0.2$ & $49 \pm 3$ & 13 & 40 & 9 \\
\hline Cy3-Et & 60 & 195 & $0.31 \pm 0$. & $14 \pm 2$ & 5 & 20 & 18 \\
\hline Cy3-H & 268 & 207 & $5.7 \pm 0.6$ & $13 \pm 4$ & 10 & 20 & 4 \\
\hline Cy5-Ph & 467 & 229 & $9.2 \pm 0.5$ & $0.028 \pm$ & 12 & 1 & 0.008 \\
\hline Cy7-Ph & 410 & - & $4.4 \pm 0.2$ & - & 11 & - & - \\
\hline Cy7-DPA & 637 & - & $2.3 \pm 0.2$ & - & 7 & - & - \\
\hline
\end{tabular}

solutions for all the monomers and aggregates across the library are shown in Table 1 (see SI Figure S3 and Table S2 for all absorption, emission spectra and lifetimes of the additional aggregates). We calculate 
the radiative rates for all the aggregates and monomers from lifetimes and quantum yield of each sample. We define excitonic superradiance $\left(\eta_{S R}\right)$ as:

$$
\eta_{S R}=\frac{\mu_{a g g}^{2}}{\mu_{m o n}^{2}}
$$

where $\mu_{a g g}$ and $\mu_{m o n}$ are the transition dipole moments of aggregate and monomer respectively. Other definitions of superradiance exist in literature, ${ }^{21,40}$ and superradiance factor as high as 62 has been reported for thiacarbocyanine J-aggregates using previous metrics. However, these do not account for the effects of the energy gap law. ${ }^{41}$ We use eq. (1) which includes the effects of drastic changes in energy gaps of aggregate to monomer using the following equation:

$$
\mu^{2}=\frac{\Phi 3 \pi \varepsilon_{o} \hbar^{4} c^{3}}{\tau n E_{g}{ }^{3}}
$$

where $\Phi, \tau, n$ and $E_{g}$ denote the quantum yield, lifetime, refractive index and energy gap respectively. We approximate the energy gap as the mean of absorption and emission peak energies.

We observe that all the Cy3 dye aggregates have significantly enhanced quantum yields at RT as compared to their respective monomers, with TDBC showing the highest $\Phi_{a g g}=49 \pm 3 \%$ (17 times higher than the monomer). On the other hand, the quantum yield of the Cy5-Ph aggregate was found to be suppressed from the monomer. Both the $\mathrm{Cy} 7$ dyes had very low quantum yields that were below our detectable limit $(<0.01 \%)$. All the Cy3 dye aggregates were also superradiant indicating enhanced transition dipole moments in the aggregates. TDBC and Cy3-Et aggregates were superradiant in spite of having higher aggregate lifetime than monomer. But the overall transition dipole moments weighted by the energy gap were still enhanced for these two aggregates as compared to their respective monomers. In case of TDBC, aggregate lifetime is 1.8 times higher than that of the monomer (without even accounting for the lower energy gap effects) but the quantum yield is about 17 times higher. Cy5-Ph aggregate had a suppressed transition dipole moment from its monomer, mainly attributed to the two orders of 
magnitude lower quantum yield than the monomer. A notable point is that according to the traditional $\mathrm{H}$ - and J-aggregate classification based on aggregate to monomer shifts, all these aggregates would be Jaggregates despite the significant differences in their emissive properties (SI Figure S3).

Temperature dependent peak shifts: To investigate the origins of such differences, we use temperature dependent spectroscopy. We stabilize all the 2D aggregates in a 50:50 (w/w) sucrose:trehalose sugar matrix, following a previously reported procedure. ${ }^{42}$ Sugar matrix stabilization protects the aggregate morphologies at low temperature so that other possibilities like the changes in the aggregate structure itself can be ruled out. Moreover, the frustrated packing afforded by structural mismatch between the sugars keep the samples optically transparent by forming a glassy matrix. Figure $3 \mathrm{~b}$ shows the absorption spectra of all J-aggregates in a sugar matrix (dashed lines) superimposed with their solution spectra (solid lines). We do not observe major changes in the lineshapes or peak positions indicating that the overall aggregate morphologies are still intact. In some cases, there is a slight broadening (Cy7-Ph) or shift (Cy5-Ph) in the sugar matrix, indicative of additional disorder though it is minor compared to the overall lineshape.

We conduct temperature dependent absorption spectroscopy on all the sugar matrix stabilized aggregates, along with temperature dependent emission for all the aggregates with detectable quantum yields. Figure $4 \mathrm{~b}-\mathrm{g}$ show one complete dataset for TDBC while those for the other dyes can be found in SI Figures S4-S7. For TDBC, we observe that the aggregate peak red shifts and broadens as we increase the temperature from $78 \mathrm{~K}$ to $300 \mathrm{~K}$ (Figure $4 \mathrm{~b}-\mathrm{c}$ ). Summarized in Table 2, all the Cy3 and Cy5 aggregates including TDBC redshift with increasing temperature while the Cy7 aggregates show blue or no shift (SI Figure S4).

The red shifting behavior for all Cy3 dyes implies a bright state that is lower in energy than the VHP. The high superradiance values (due to enhancement of radiative rates) suggest that they are not only at 
lower energy than the VHP but are also at the band edge. Therefore, we classify all the Cy3 aggregates including TDBC as Kasha's J-aggregates with band edge bright states. In contrast, Cy5-Ph aggregates have a red shifting peak with temperature even though the radiative rate is suppressed from the monomer as indicated by the $\eta_{S R}<1$. This implies that the bright state of Cy5-Ph is at lower energy of the VHP but still has significant dark states below such that non-radiative pathways dominate the excited state decay. Therefore, we classify Cy5-Ph as an I-aggregate with a bright state lower in energy than the VHP. All the Cy3 and Cy5 aggregates show similar trends in temperature dependent emission as well, where the aggregate peaks red shift with increasing temperature (SI Figures S6-S7).
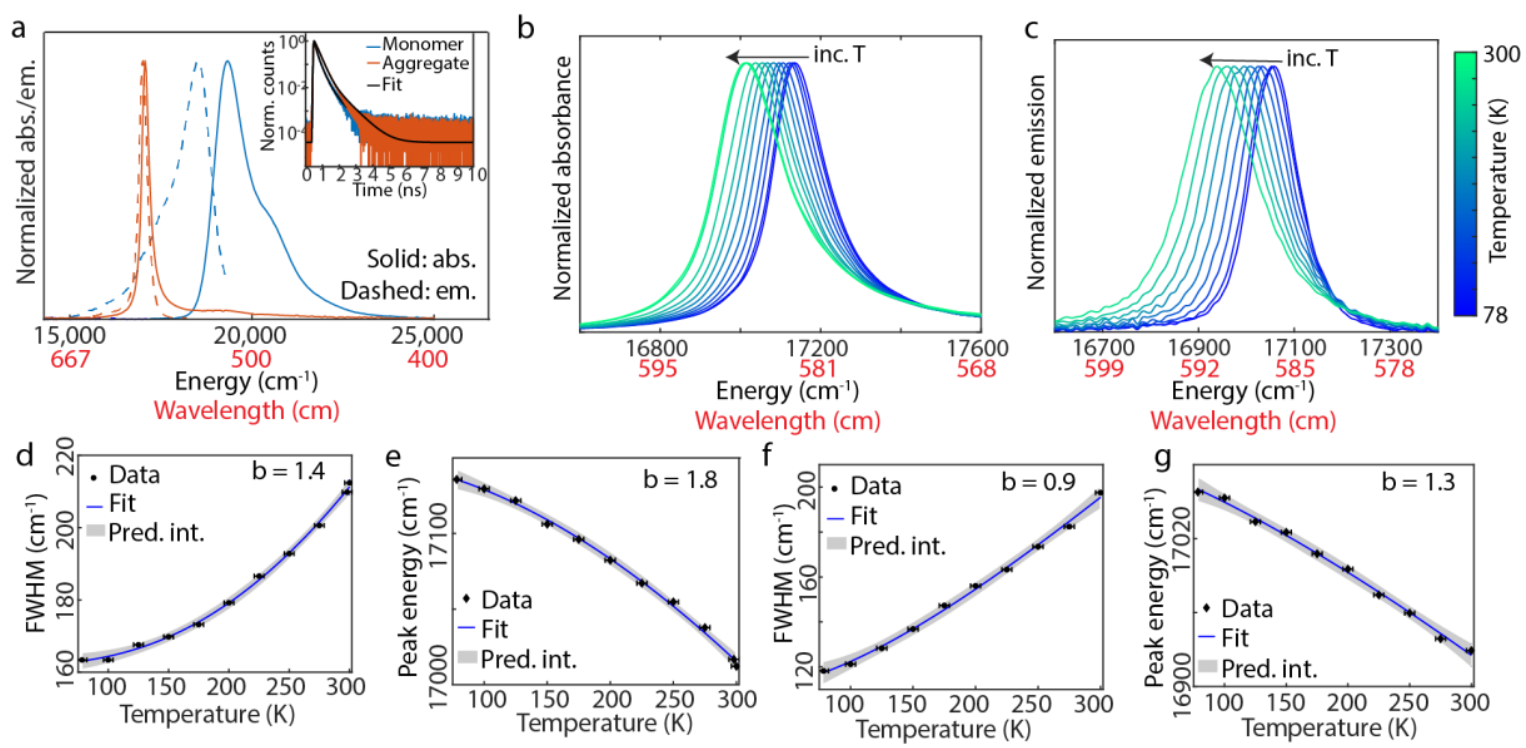

Figure 4. a. Normalized absorption (solid lines) and emission (dashed lines) spectra for TDBC monomer (blue), and TDBC aggregate (red). Inset: Time correlated single photon counting data for TDBC monomer (blue), and TDBC aggregate (red) with biexponential fits in black, b-g. temperature dependent spectroscopy data for TDBC aggregate in sugar matrix from $78 \mathrm{~K}$ to $300 \mathrm{~K}$, b. normalized absorption spectra, c. normalized emission spectra, d. absorption full-width at half-maximum (FWHM), e. absorption peak energy, f. emission FWHM, g. emission peak energy. Fits are from eq. (3) and (4) for FWHM and peak energies, respectively. Error bars are estimates of $\pm 2 \mathrm{~K}$.

Recalling Figure 2, the temperature dependent shifts are sensitive to the local slope of the DOS. ${ }^{31}$

Therefore, Cy7-Ph which showed no shift (SI Figure S4), must have a bright state farther from the bandedge in a relatively flat region between the VHPs. Since Cy7-DPA is blue shifting with temperature, it has 
a bright state at higher energy than the VHP (or locally negative slope of the DOS). Therefore, we classify both Cy7 cases as I-aggregates.

These observations are also consistent with our previous studies where we used a thermal broadening based metric to conclude that both $\mathrm{Cy} 7$ aggregates were I-aggregates. Further, Cy7-Ph has a bright state farther away from the band edge as compared to Cy7-DPA. ${ }^{22}$ The relative distance of the bright state from the band edge can be qualitatively gauged by means of the amount of thermal broadening. In cases where the bright state so far from the band edge that it is thermally inaccessible, one expects no broadening (irrespective of the relative position to the VHP), as in the Cy7-Ph aggregate (SI Figure S4). Cy7-DPA shows thermal broadening despite being an I-aggregate, meaning that the bright state is relatively closer to the band edge.

Temperature dependent power laws: In band-edge J-aggregates, thermal line broadening and shifts are modeled using exciton-phonon coupling with the environment, previously described with power laws which uniquely reflect the combination of exciton DOS and exciton-phonon coupling spectral density (also called as system-bath coupling). $\cdot{ }^{43-47}$ Linewidths can be thought of as the sum of rates of various phonon assisted exciton scattering processes. The rates are a result of energy conservation between the system and the surrounding bath, as well as the couplings between the two. The latter is a constant for homogenous, translationally symmetric systems while the former gives a weighted average of systembath DOS, and therefore the power law dependence. Such power law scalings of FWHM and peak shifts can be used as an indicator for the types of underlying exciton-phonon coupling processes. We fit the extracted linewidths and peak energies to power laws as shown in Figure 4d-g. The following eq. (3) and eq. (4) were used for fitting the FWHM $(\omega)$ and peak energies $\left(v_{o}\right)$, respectively:

$$
\begin{gathered}
\omega^{2}=\left(a T^{b}\right)^{2}+c^{2} \\
v_{0}=a_{0} T^{b_{0}}+c_{0}
\end{gathered}
$$


The first terms denote the homogeneous contributions following a power law $\left(b, b_{0}\right.$ for FWHM and peak energies, respectively) while the second term ( $c, c_{0}$ for FWHM and peak energies, respectively) denotes the extrapolated inhomogeneous limit at $0 \mathrm{~K}$ (details in SI Section 3). Table 2 summarizes the power laws obtained from temperature dependent absorptions for all band-edge aggregates. The corresponding power law fits for the rest of the aggregates are shown in SI Figure S5.

Table 2. Summary of temperature dependent absorption data for 2D aggregates. Power laws (b parameter from eq. (3) for FWHM and $b_{0}$ from eq. (4) for peak energies), and direction of temperature dependent peak shifts. Errors are from $95 \%$ confidence intervals.

\begin{tabular}{|c|c|c|c|}
\hline \multirow{2}{*}{ Dye } & Peak shift with & Power law for & Power law for peak \\
& temperature & FWHM $(\boldsymbol{b})$ & energies $\left(\boldsymbol{b}_{\boldsymbol{0}}\right)$ \\
\hline TDBC & Red & $1.4 \pm 0.2$ & $1.8 \pm 0.3$ \\
\hline Cy3-Et & Red & $0.9 \pm 0.1$ & $1.0 \pm 0.2$ \\
\hline Cy3-H & Red & $1.0 \pm 0.2$ & $1.1 \pm 0.6$ \\
\hline Cy5-Ph & Red & $1.0 \pm 0.2$ & $1.1 \pm 0.3$ \\
\hline Cy7-Ph & None & - & - \\
\hline Cy7-DPA & Blue & - & - \\
\hline
\end{tabular}

Despite similar excitonic shifts and energies, we find that the benzathiazole aggregates display a different power-law dependence in its spectral shift and FWHM compared to the benzimidazole dye TDBC aggregate. We hypothesize that the different heterocycles and additional alkyl group result in a modified phonon DOS for TDBC. The power laws observed here for 2D aggregates are lower than those reported for other 1D and quasi-1D systems such as light-harvesting nanotubes ( $b=2 \pm 0.5)$ and pseudoisocyanine $(b=3.4)^{42,46}$ 
Changes in linewidths arise from the coupling of exciton degrees of freedom (system) to vibrational degrees of freedom (environment/ phonon bath). Specifically, under the weak-coupling, fast-environment limit, the power-law exponent $(b)$ of the temperature dependent line width can be broken down as $b=$ $d+s+1$, where $d$ and $s$ are the scaling of the exciton DOS at the band edge, and low-frequency systembath coupling spectral density respectively. ${ }^{43,44,48} s=1$ for an Ohmic bath while $s<1$ for a subohmic bath. A similar expression can be derived for temperature dependent peak shifts which reflects the same underlying band structure (see SI Section 3). ${ }^{31}$ For 2D excitonic systems with non-nearest neighbor dipoledipole couplings, the system DOS scaling has been shown to follow a $d=0.5$ dependence. ${ }^{49}$ Thus, we obtain $s=0$ for TDBC and $s \sim-0.5$ for the rest of the benzothiazole Cy3 and Cy5 aggregates indicating that the system-bath coupling spectral density in the TDBC aggregate is independent of frequency while the negative sign for rest of the band-edge aggregates indicates an inverse scaling. The scaling laws may be explained by the fact that the excitonic couplings in these systems are quite large $\left(\sim 10^{3} \mathrm{~cm}^{-1}\right)$, and the higher frequency components of system-bath coupling will be highly suppressed in comparison. For finite coherent length, pure dephasing may compete with population relaxation and lead to linear scaling with temperature. ${ }^{48}$ Although, this is unlikely in our case, since all aggregates had up to micron scale sizes as seen from cryoEM images (SI Figure S2). ${ }^{36}$

Screening aggregate geometries using stochastic modeling of the Hamiltonian: In Figure 5a-b, we show the exciton bandwidth and position of $k=0$ bright state as a function of slip for zero disorder case for different dipolar coupling models, obtained using the procedure in SI Section 2. We observe significant differences in the slip dependent properties of the excitonic band structure depending on the coupling model as well as the aggregate packing geometry. The use of an extended dipole model opens up a larger range of slips which show l-aggregate behavior. We attribute this to the point dipole model that shows a steeper slip dependence underestimating the short range couplings (as seen in SI Figure S1b) and therefore, results in a very small I-aggregate region. Further, the slip dependence for Cy7-DPA (Figure 5c) 
shows the effect of longer brick length, where the possible slip range for I-aggregation is expanded further.

This is the result of increased positive contributions to the short-range couplings due to longer molecular species. ${ }^{50}$ The total band width of the excitonic DOS is bounded by twice the absolute value of the net coupling which has a minimum at zero i.e. when it changes sign from positive to negative. As a result, the exciton bandwidth is minimized around the boundary between I- and J-aggregate regions.
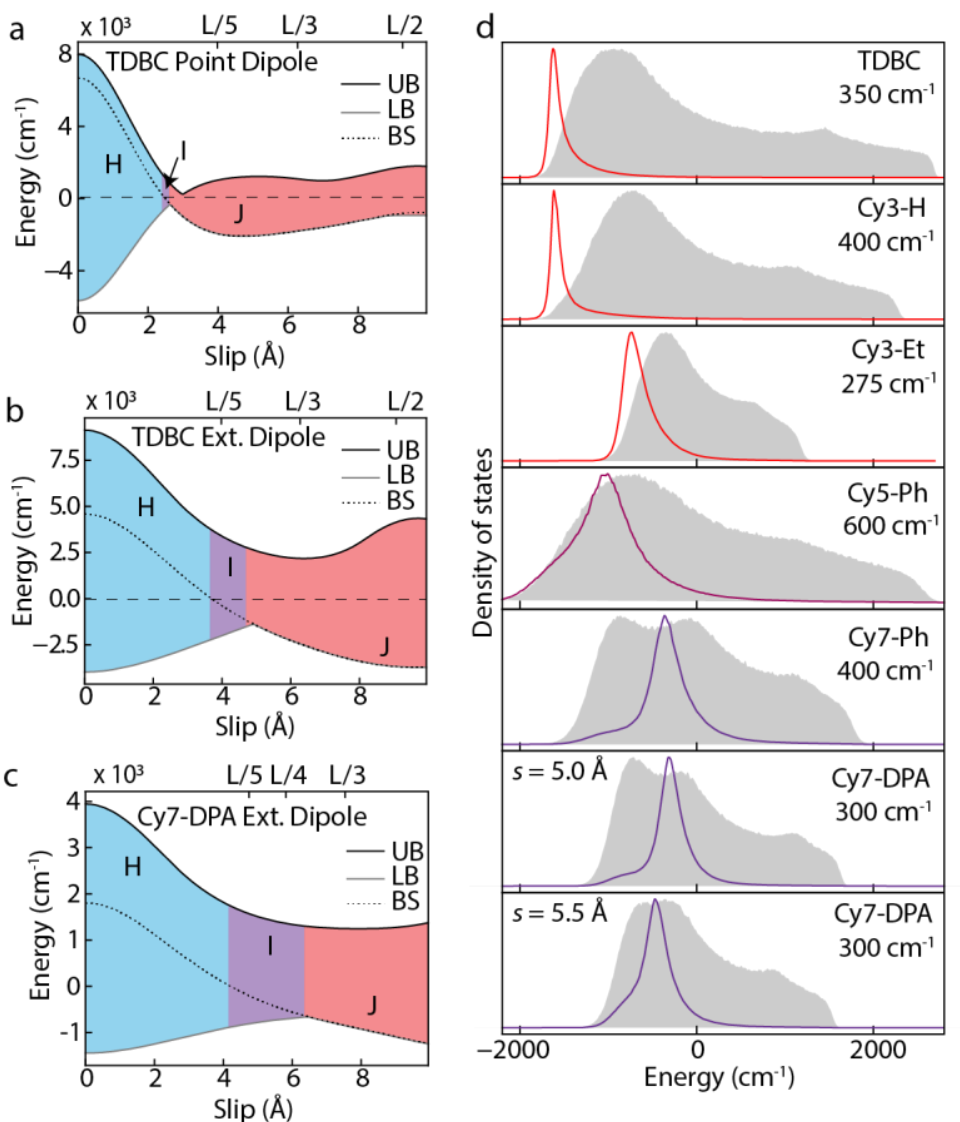

e
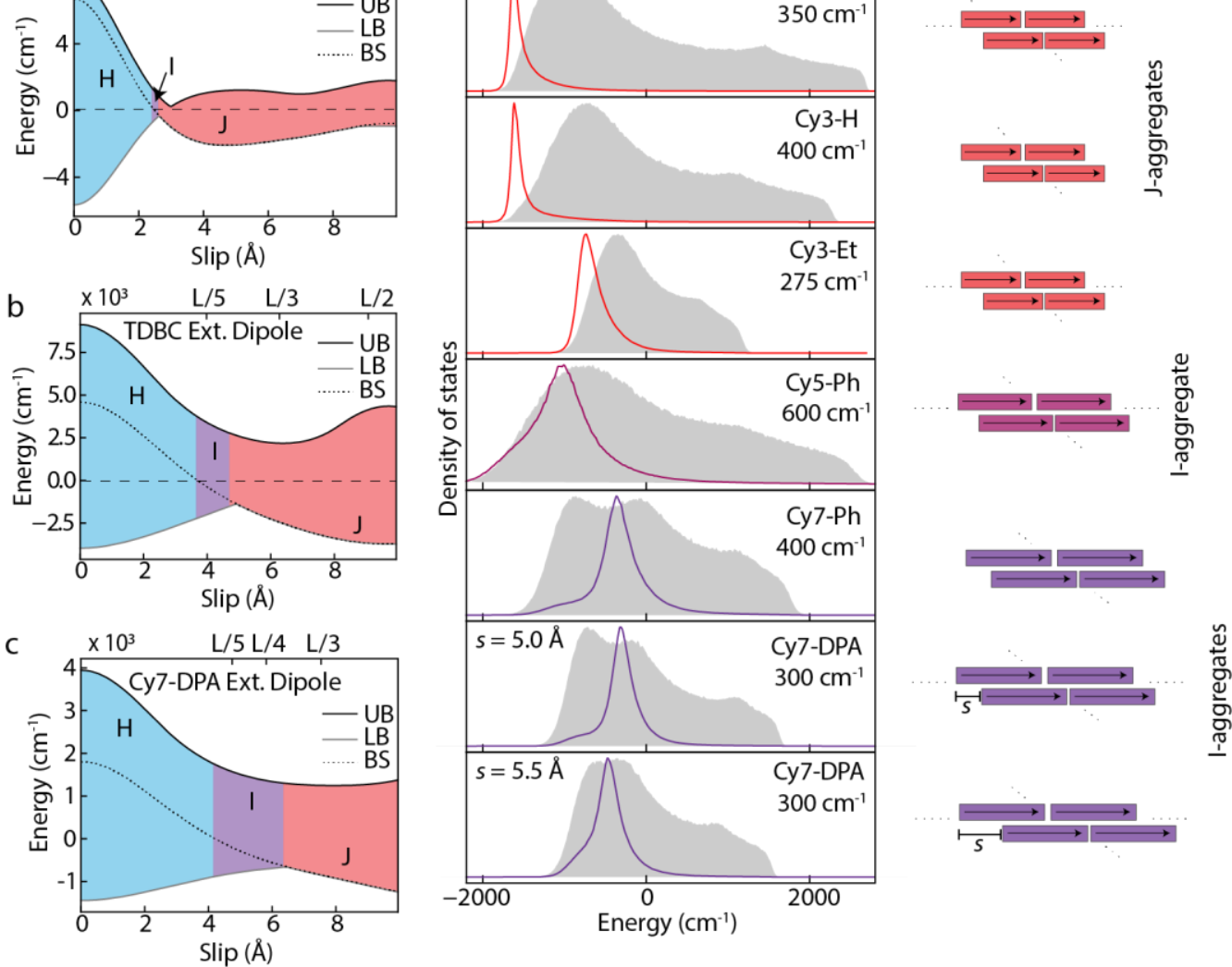

Figure 5. a-c. Slip dependent behavior of the band structure- upper band edge (UB, black line), lower band edge (LB, grey line) and bright state (BS, dotted line) with $\mathrm{H}-$-, I- and J-aggregation regions color coded as blue, violet and red respectively for 2D aggregates of a. TDBC using point dipole model, b. TDBC using extended dipole model, $\mathrm{c}$. Cy7-DPA using extended dipole model. Dashed lines indicate monomer energy and dotted lines indicate the bright state, $d$. calculated excitonic density of states (grey region) and absorption spectra (colored lines) at $0 \mathrm{~K}$ for all $2 \mathrm{D}$ aggregates with a slip of $5.0 \AA$ (top six panels) and given disorder values. The last panel shows the same for Cy7DPA with higher slip of $5.5 \AA$, e. schematics of the corresponding brick layer geometries.

We screen large parameter space of possible aggregate packing parameters (slips) and disorder values using the stochastic sampling methods for the DOS, and Chebyshev kernels the absorption spectra, following Bradbury et al. ${ }^{23}$ The stochastic density of states is calculated by taking a random superposition 
of all eigenstates, projecting it down onto a specific energy in the spectrum, and then averaging over many random samplings. This approach is computationally less expensive than diagonalizing large Hamiltonians and allows for rapid screening of several aggregate geometries with realistic sizes ( $10^{6}$ monomer units). Several disorder realizations can be afforded at no additional cost. By screening thousands of configurations for each aggregate, we obtain the slip dependence of the excitonic band and the position of the bright state $(k=0)$. In SI Figure S8, we show large parameter screens for FWHM and peak positions of all six aggregates. Best fit slip parameters for each aggregate were determined by comparing the calculated spectral widths to the FWHM at $0 \mathrm{~K}$ obtained from the power law fits listed in Table S1 i.e parameter $c$ from eq. (3). We narrow down the possible slip - disorder space to the grey squares in SI Figure S8.

From the narrowed down range of slips (grey squares on Figure S8), we use a slip of $5.0 \AA$ and plot the calculated excitonic DOS and absorption spectra in Figure $5 \mathrm{~d}$. While the realistic slips may be slightly different for each aggregate depending on the central position substitution, we use this representative value as it was consistent with our experimental observations among the narrowed down range of slips. As the brick length increases from Cy3 to Cy7 dyes and relative slip (normalized to brick length) decreases (Figure 5e), we observe that the exciton band structure goes from band-edge J-aggregate to mid-band Iaggregate. Our calculations in Figure $5 \mathrm{~d}$ show that all the Cy3 dyes have a band-edge bright state, lower energy than the VHP. This is consistent with our inference from Tables 1 and 2, based on temperature dependent shifts and emissive properties. Cy5-Ph, while lower energy than the VHP, also has a broad linewidth with significant oscillator strength farther up in the DOS, explaining the suppression of aggregate to monomer quantum yield and the red shifting behavior. Finally, Cy7-Ph and Cy7-DPA both have bright states away from the band-edge, explaining the I-aggregate behavior seen in our experiments. Cy7-Ph also has the absorption peak in the relatively flat region of the DOS, consistent with our temperature dependent experiments which showed no shift. While Cy7-DPA appears to have local positive slope at 5.0 
$\AA$ slip, the realistic slip is higher than that of Cy7-Ph due to a bulkier center position substitution as we have previously shown. ${ }^{22}$ This is shown in the bottom two panels of Figure $5 d$, where a higher slip value pushes the bright state in a locally negative slope region of the DOS, explaining the temperature dependent blue shifts. The bottom three panels clearly confirm our inference from Table 2 that both the Cy7 aggregates are l-aggregates.

Thus, supramolecular modulation of chromophore packing in 2D aggregates can allow for tunable excitonic band structures where the position of the bright state relative to the band-edge and to the VHP can be controlled with slips and molecular lengths. Such dependencies are a direct consequence of the relative contributions of positive and negative couplings afforded by different brick layer geometries. Furthermore, these relations can be exploited to chemically tune the band structures of higher dimensional aggregates and ultimately control the photophysical properties relevant for their applications. J-aggregates can be useful for non-invasive SWIR imaging due to their high quantum yields and narrow linewidths but SWIR aggregates reported so far have very low quantum yields $\left(\phi_{F}<1 \%\right)$, possibly because many are, in fact, non-band edge I-aggregates. ${ }^{11,12}$ By making SWIR aggregates with higher slips, their bright states can be pushed close to the band-edge yielding high quantum yield Jaggregates in SWIR, which could be transformative for SWIR technologies.

We note that our findings are agnostic of the nature of the couplings (dipole-dipole, charge- transfer etc.) and depend mainly on the relative strengths of the short and long range couplings. Here, we show the importance of supramolecular packing parameter in modulating the relative contributions of short and long range couplings. In addition to the supramolecular geometries, the type of the nearest-neighbor couplings can also be used to modulate the relative contributions from short and long range couplings. Specifically, the point dipole model greatly overestimates the short-range contributions and results in a very small I-aggregate region while the more accurate TC model gives a much larger I-aggregate region for the same geometric parameters (Figure 5a-b). 


\section{Conclusions}

Our work shows that different nanoscale aggregate geometries can be used to avail the vast diversities in the excitonic properties of 2D aggregates. Excitonic band structure of such aggregates can be experimentally probed using temperature dependent spectroscopy, which forms a comprehensive tool, relating specific experimental observables to distinct aspects of the excitonic band structure. We show temperature dependence studies of six 2D aggregates where carefully chosen dyes with varying brick lengths and central position substitutions afford the different aggregate geometries. Different nearest neighbor coupling curves could be realized with charge-transfer interactions (e.g. donor acceptor molecules). ${ }^{19,20}$

Photophysical properties are highly dependent on the position of the bright state within the DOS. Jaggregates, with a band edge bright state, are known to have high quantum yields and superradiance rates, $^{1,14,40}$ making them applicable in several areas such as shortwave infrared imaging, ${ }^{11,12}$ polaritonics, ${ }^{8}$ and telecommunications. ${ }^{13} \mathrm{~J}$-aggregates have also been shown to exhibit fast picosecond FRET, serving as efficient antennas and bridges for energy transfer. ${ }^{3,51}$ I-aggregates on the other hand have low quantum yields. Yet, the mid band bright state can exhibit collective oscillations of transition dipoles, enabling fast energy or charge transfer in the near-field. ${ }^{22,52}$ Supramolecular tunability of the excitonic band structure, demonstrated here, will therefore have exciting implications in several fields including shortwave infrared imaging, excitonic energy transfer, polaritonics, and plexitonics. ${ }^{5,53}$

\section{Notes}

*Corresponding author

Email: jcaram@chem.ucla.edu

No conflict of interest. 


\section{Acknowledgements}

This work was supported by NSF CHE grant no. 1905242 and Faculty Research Grant (UCLA Academic Senate). NCB and DN are grateful for support by the NSF grant CHE-1763176. APD thanks UCLA Graduate Division Dissertation Year Fellowship for financial support. NG is grateful for a PROMOS stipend granted by Leipzig University, Germany. NCB thanks the NSF-GRFP under grant no. DGE-2034835. Cao is supported by NSF (CHE 1800301 and CHE 1836913). APD and JRC thank Prof. Benjamin J. Schwartz, UCLA for helpful discussions. APD thanks Ray Huang for formatting SI figures. Authors acknowledge the Materials Characterization Laboratory, UCLA Chemistry \& Biochemistry for instrumentation.

\section{References}

(1) Bricks, J. L.; Slominskii, Y. L.; Panas, I. D.; Demchenko, A. P. Fluorescent J-Aggregates of Cyanine Dyes: Basic Research and Applications Review. Methods Appl. Fluoresc. 2017, 6 (1), 012001.

(2) Brixner, T.; Hildner, R.; Köhler, J.; Lambert, C.; Würthner, F. Exciton Transport in Molecular Aggregates - From Natural Antennas to Synthetic Chromophore Systems. Adv. Energy Mater. 2017, 7 (16), 1700236.

(3) Wang, C.; Weiss, E. A. Sub-Nanosecond Resonance Energy Transfer in the Near-Infrared within Self-Assembled Conjugates of PbS Quantum Dots and Cyanine Dye J-Aggregates. J. Am. Chem. Soc. 2016, $138(30)$, 9557-9564.

(4) Oh, J. W.; Kumazaki, S.; Rubtsov, I. V.; Suzumoto, T.; Tani, T.; Yoshihara, K. Ultrafast Energy Transfer in J-Aggregate on AgBr Microcrystals: Its Dependence on Dye Coverage. Chem. Phys. Lett. 2002, 352 (5-6), 357-362.

(5) Saikin, S. K.; Eisfeld, A.; Valleau, S.; Aspuru-Guzik, A. Photonics Meets Excitonics: Natural and Artificial Molecular Aggregates. Nanophotonics 2013, 2 (1), 21-38. 
(6) Kriete, B.; Feenstra, C. J.; Pshenichnikov, M. S. Microfluidic Out-of-Equilibrium Control of Molecular Nanotubes. Phys. Chem. Chem. Phys. 2020, 22 (18), 10179-10188.

(7) Gibbs, H. M.; Khitrova, G.; Koch, S. W. Exciton-Polariton Light-Semiconductor Coupling Effects. Nat. Photonics 2011, 5 (5), 273-273.

(8) Zhong, X.; Chervy, T.; Wang, S.; George, J.; Thomas, A.; Hutchison, J. A.; Devaux, E.; Genet, C.; Ebbesen, T. W. Non-Radiative Energy Transfer Mediated by Hybrid Light-Matter States. Angew. Chemie - Int. Ed. 2016, 55 (21), 6202-6206.

(9) Eisfeld, A.; Briggs, J. S. Dye Aggregates in Luminescent Solar Concentrators. Phys. Status Solidi Appl. Mater. Sci. 2018, 215 (2).

(10) Pozin, S. I.; Lypenko, D. A.; Perelygina, O. M.; Gribkova, O. L.; Prokhorov, V. V.; Mal'tsev, E. I. Polymer Composite with J-Aggregates of Polymethine Dye as a Charge-Transport Layer of Organic Light-Emitting Diode. Inorg. Mater. Appl. Res. 2021121 2021, 12 (1), 94-100.

(11) Chen, W.; Cheng, C. A.; Cosco, E. D.; Ramakrishnan, S.; Lingg, J. G. P.; Bruns, O. T.; Zink, J. I.; Sletten, E. M. Shortwave Infrared Imaging with J-Aggregates Stabilized in Hollow Mesoporous Silica Nanoparticles. J. Am. Chem. Soc. 2019, 141 (32), 12475-12480.

(12) Sun, C.; Li, B.; Zhao, M.; Wang, S.; Lei, Z.; Lu, L.; Zhang, H.; Feng, L.; Dou, C.; Yin, D.; Xu, H.; Cheng, Y.; Zhang, F. J-Aggregates of Cyanine Dye for NIR-II in Vivo Dynamic Vascular Imaging beyond 1500 Nm. J. Am. Chem. Soc. 2019, 141 (49), 19221-19225.

(13) Bouit, P. A.; Wetzel, G.; Berginc, G.; Loiseaux, B.; Toupet, L.; Feneyrou, P.; Bretonnière, Y.; Kamada, K.; Maury, O.; Andraud, C. Near IR Nonlinear Absorbing Chromophores with Optical Limiting Properties at Telecommunication Wavelengths. Chem. Mater. 2007, 19 (22), 5325-5335.

(14) Doria, S.; Sinclair, T. S.; Klein, N. D.; Bennett, D. I. G.; Chuang, C.; Freyria, F. S.; Steiner, C. P.; 
Foggi, P.; Nelson, K. A.; Cao, J.; Aspuru-Guzik, A.; Lloyd, S.; Caram, J. R.; Bawendi, M. G.

Photochemical Control of Exciton Superradiance in Light-Harvesting Nanotubes. ACS Nano 2018, $12(5), 4556-4564$.

(15) Kasha, M. Energy Transfer Mechanisms and the Molecular Exciton Model for Molecular Aggregates. Radiat. Res. 1963, 20 (1), 55-71.

(16) Kasha, M.; Rawls, H. R.; El-Bayoumi, M. A. The Exciton Model In Molecular Spectroscopy. Pure Appl. Chem. 1965, 11 (3-4), 371-392.

(17) Scheblykin, I. G.; Bataiev, M. M.; Van der Auweraer, M.; Vitukhnovsky, A. G. Dimensionality and Temperature Dependence of the Radiative Lifetime of J-Aggregates with Davydov Splitting of the Exciton Band. Chem. Phys. Lett. 2000, 316 (1-2), 37-44.

(18) Knapp, E. W. Lineshapes of Molecular Aggregates, Exchange Narrowing and Intersite Correlation. Chem. Phys. 1984, 85 (1), 73-82.

(19) Bialas, D.; Zhong, C.; Würthner, F.; Spano, F. C. Essential States Model for Merocyanine Dye Stacks: Bridging Electronic and Optical Absorption Properties. J. Phys. Chem. C 2019, 123 (30), 18654-18664.

(20) Zheng, C.; Zhong, C.; Collison, C. J.; Spano, F. C. Non-Kasha Behavior in Quadrupolar Dye Aggregates: The Red-Shifted H-Aggregate. J. Phys. Chem. C 2019, 123 (5), 3203-3215.

(21) Eisfeld, A.; Marquardt, C.; Paulheim, A.; Sokolowski, M. Superradiance from Two Dimensional Brick-Wall Aggregates of Dye Molecules: The Role of Size and Shape for the Temperature Dependence. Phys. Rev. Lett. 2017, 119 (9), 097402.

(22) Deshmukh, A. P.; Koppel, D.; Chuang, C.; Cadena, D. M.; Cao, J.; Caram, J. R. Design Principles for Two-Dimensional Molecular Aggregates Using Kasha's Model: Tunable Photophysics in Near and 
Short-Wave Infrared. J. Phys. Chem. C 2019, 123 (30), 18702-18710.

(23) Bradbury, N. C.; Chuang, C.; Deshmukh, A. P.; Rabani, E.; Baer, R.; Caram, J. R.; Neuhauser, D. Stochastically Realized Observables for Excitonic Molecular Aggregates. J. Phys. Chem. A 2020, 124 (49), 10111-10120.

(24) Yamagata, H.; Spano, F. C. Interplay between Intrachain and Interchain Interactions in Semiconducting Polymer Assemblies: The HJ-Aggregate Model. J. Chem. Phys. 2012, 136 (18), 184901.

(25) Clark, J.; Silva, C.; Friend, R. H.; Spano, F. C. Role of Intermolecular Coupling in the Photophysics of Disordered Organic Semiconductors: Aggregate Emission in Regioregular Polythiophene. Phys. Rev. Lett. 2007, 98 (20), 206406.

(26) Spano, F. C. Analysis of the UV/Vis and CD Spectral Line Shapes of Carotenoid Assemblies: Spectral Signatures of Chiral H-Aggregates. J. Am. Chem. Soc. 2009, 131 (12), 4267-4278.

(27) Hestand, N. J.; Spano, F. C. Expanded Theory of H- and J-Molecular Aggregates: The Effects of Vibronic Coupling and Intermolecular Charge Transfer. Chem. Rev. 2018, 118 (15), 7069-7163.

(28) Didraga, C.; Pugžlys, A.; Hania, P. R.; von Berlepsch, H.; Duppen, K.; Knoester, J. Structure, Spectroscopy, and Microscopic Model of Tubular Carbocyanine Dye Aggregates. J. Phys. Chem. B 2004, 108 (39), 14976-14985.

(29) Freyria, F. S.; Cordero, J. M.; Caram, J. R.; Doria, S.; Dodin, A.; Chen, Y.; Willard, A. P.; Bawendi, M. G. Near-Infrared Quantum Dot Emission Enhanced by Stabilized Self-Assembled J-Aggregate Antennas. Nano Lett. 2017, 17 (12), 7665-7674.

(30) Wang Li-Li Li Editors, H. Nanomedicine and Nanotoxicology In Vivo Self-Assembly Nanotechnology for Biomedical Applications. 
(31) Chuang, C.; Bennett, D. I. G.; Caram, J. R.; Aspuru-Guzik, A.; Bawendi, M. G.; Cao, J. Generalized Kasha's Model: T-Dependent Spectroscopy Reveals Short-Range Structures of 2D Excitonic Systems. Chem 2019, 5 (12), 3135-3150.

(32) Czikklely, V.; Forsterling, H. D.; Kuhn, H. Extended Dipole Model for Aggregates of Dye Molecules. Chem. Phys. Lett. 1970, 6 (3), 207-210.

(33) Ghosh, B.; Mardanya, S.; Singh, B.; Zhou, X.; Wang, B.; Chang, T.-R.; Su, C.; Lin, H.; Agarwal, A.; Bansil, A. Saddle-Point Van Hove Singularity and Dual Topological State in $\$\{\backslash r m$ Pt $\}\{2\}\{\backslash r m$ HgSe\}_\{3\}\$. Phys. Rev. B 2019, 100, 235101.

(34) Xing, D. Y.; Liu, M.; Gong, C. D. Comment on Anomalous Isotope Effect and van Hove Singularity in Superconducting Cu Oxides. Physical Review Letters. 1992, p 1090.

Li, G.; Luican, A.; Lopes Dos Santos, J. M. B.; Castro Neto, A. H.; Reina, A.; Kong, J.; Andrei, E. Y. Observation of Van Hove Singularities in Twisted Graphene Layers. Nat. Phys. 2009.

Deshmukh, A. P.; Bailey, A. D.; Forte, L. S.; Shen, X.; Geue, N.; Sletten, E. M.; Caram, J. R. Thermodynamic Control over Molecular Aggregate Assembly Enables Tunable Excitonic Properties across the Visible and Near-Infrared. J. Phys. Chem. Lett. 2020, 11 (19), 8026-8033.

Moll, J.; Daehne, S.; Durrant, J. R.; Wiersma, D. A. Optical Dynamics of Excitons in J Aggregates of a Carbocyanine Dye. J. Chem. Phys. 1995, 102 (16), 6362-6370.

(38)

Sorokin, A. V.; Ropakova, I. Y.; Grynyov, R. S.; Vilkisky, M. M.; Liakh, V. M.; Borovoy, I. A.; Yefimova, S. L.; Malyukin, Y. V. Strong Difference between Optical Properties and Morphologies for J-Aggregates of Similar Cyanine Dyes. Dye. Pigment. 2018, 152, 49-53.

Müller, M.; Paulheim, A.; Eisfeld, A.; Sokolowski, M. Finite Size Line Broadening and Superradiance of Optical Transitions in Two Dimensional Long-Range Ordered Molecular 
Aggregates. J. Chem. Phys. 2013, 139 (4), 044302.

(40) Özçelik, S.; Akins, D. L. Superradiance of Aggregated Thiacarbocyanine Molecules. J. Phys. Chem. B 1999, 103 (42), 8926-8929.

(41) Friedman, H. C.; Cosco, E. D.; Atallah, T. L.; Sletten, E. M.; Caram, J. R. Establishing Design Principles for Emissive Organic SWIR Chromophores from Energy Gap Laws. ChemRxiv 2020.

(42) Caram, J. R.; Doria, S.; Eisele, D. M.; Freyria, F. S.; Sinclair, T. S.; Rebentrost, P.; Lloyd, S.; Bawendi, M. G. Room-Temperature Micron-Scale Exciton Migration in a Stabilized Emissive Molecular Aggregate. Nano Lett. 2016, 16 (11), 6808-6815.

(43) Heijs, D. J.; Malyshev, V. A.; Knoester, J. Thermal Broadening of the J-Band in Disordered Linear Molecular Aggregates: A Theoretical Study. J. Chem. Phys. 2005, 123 (14), 144507.

(44) Heijs, D. J.; Malyshev, V. A.; Knoester, J. Decoherence of Excitons in Multichromophore Systems: Thermal Line Broadening and Destruction of Superradiant Emission. Phys. Rev. Lett. 2005, 95 (17), 177402.

(45) Mattoon, R. W. Polymer Spectra of a Cyanine Dye. J. Chem. Phys. 2004, 12 (6), 268.

(46) Renge, I.; Wild, U. P. Solvent, Temperature, and Excitonic Effects in the Optical Spectra of Pseudoisocyanine Monomer and J-Aggregates. J. Phys. Chem. A 1997, 101 (43), 7977-7988.

(47) Scheblykin, I. G.; Sliusarenko, O. Y.; Lepnev, L. S.; Vitukhnovsky, A. G.; Auweraer, M. Van Der. Excitons in Molecular Aggregates of 3,3'-Bis-[3-Sulfopropyl]-5,5'-Dichloro-9-

Ethylthiacarbocyanine (THIATS): Temperature Dependent Properties. J. Phys. Chem. B 2001, 105, 4636-4646.

(48) Dijkstra, A. G.; Duan, H.-G.; Knoester, J.; Nelson, K. A.; Cao, J. How Two-Dimensional Brick Layer J- 
Aggregates Differ from Linear Ones: Excitonic Properties and Line Broadening Mechanisms. J.

Chem. Phys. 2016, 144 (13), 134310.

(49) Chuang, C.; Cao, J. Universal Scalings in Two-Dimensional Anisotropic Dipolar Excitonic Systems. Phys. Rev. Lett. 2021, 127.

(50) Brent P. Krueger; Gregory D. Scholes, and; Fleming*, G. R. Calculation of Couplings and EnergyTransfer Pathways between the Pigments of LH2 by the Ab Initio Transition Density Cube Method. J. Phys. Chem. B 1998, 102 (27), 5378-5386.

(51) Wang, C.; Weiss, E. A. Accelerating FRET between Near-Infrared Emitting Quantum Dots Using a Molecular J-Aggregate as an Exciton Bridge. Nano Lett. 2017, 17 (9), 5666-5671.

(52) Gao, X.; Eisfeld, A. Near-Field Spectroscopy of Nanoscale Molecular Aggregates. J. Phys. Chem. Lett. 2018, 9 (20), 6003-6010.

(53) Chevrier, K.; Benoit, J. M.; Symonds, C.; Paparone, J.; Laverdant, J.; Bellessa, J. Organic Exciton in Strong Coupling with Long-Range Surface Plasmons and Waveguided Modes. ACS Photonics 2018, $5(1), 80-84$. 